\section{Conclusion}

In conclusion it would appear desirable to measure the wave length shift associated with thermal spike scattering and corroborate the application of the impulse approximation to high angle scattering from amorphous media. Such high angle scattering could also be used to measure the scattering factors for core electrons of heavy atoms (for example $\mathrm{Hg}$ as a liquid) where relativistic effects are important. In addition thermal spike scattering can be used to standardize Compton scattering since the "elastic"

1 R. Currat, P. D. DeCicco, and R. J. Weiss, Phys. Rev. 4, 4256 [1971]. component can be accurately calculated from Hartree-Fock free atom core scattering factors. Lastly such thermal spike scattering may be followed by emission of a photon and this might provide a convenient method to study these localized excitations. By employing W $\mathrm{K} \alpha$ instead of $\mathrm{Ag} \mathrm{K} \alpha$ the thermal spike energy and the angular shift in the analyzer are increased by almost a factor of 10 . Under these conditions one might even measure an atomic "Compton" profile and determine the atomic momentum distributions.

2 P. Eisenberger and P. Platzmann, Phys. Rev. A 2, 415 [1970].

3 W. C. Phillips and R. J. Weiss, Phys. Rev. 111, 790 [1968].

\title{
Elastic Scattering of Bloch Waves in Absorbing Crystals Containing Lattice Defects
}

\author{
M. Wilkens, K.-H. Katerbau, and M. Rühle \\ Max-Planck-Institut für Metallforschung, Stuttgart, Germany \\ (Z. Naturforsch. 28 a, 681-690 [1973] ; received 22 January 1973) \\ Dedicated to Professor Dr. G. Borrmann on his 65th birthday
}

\begin{abstract}
The differential equations of Bloch wave type which are used in transmission electron micro scopy for the calculation of the diffraction contrast from lattice defects are discussed with special reference to crystals with anomalous absorption (i. e. with a complex crystal potential). anomalous absorption is included, the eigenvalue equation by which the Bloch waves are defined in the perfect reference lattice, becomes non-Hermitian. It is shown that for some particular contrast effects not only the eigenvalues but also the eigenvectors must be corrected for the imaginary part of the crystal potential. As a consequence the orthogonality of the Bloch waves is violated. The correction of the eigenvectors may result in long-range contrast tails of the images of lattice defects (intraband scattering). If, however, only the peak positions of the contrast profiles are considered, it suffices in practical cases to correct only the eigenvalues as is usually assumed for the diffraction in perfect crystals.
\end{abstract}

\section{Introduction}

The elastic scattering of fast electrons in crystals can be described conveniently assuming a complex crystal potential. In transmission electron microscopy of crystalline specimens this was first demonstrated by Hashimoto, Howie and Whelan ${ }^{1}$. While the real part of the potential is responsible for the elastic diffraction of the electrons into the Braggreflexions the imaginary part accounts for inelastic scattering processes which lead to an attenuation of the wave functions of the elastically scattered electrons by absorption. One of the most striking phenomena which was successfully explained by such a complex crystal potential is the anomalous absorp-

Reprint requests to Dr. M. Wilkens, Max-Planck-Institut für Metallforschung, D-7000 Stuttgart, Azenbergstr. 12. tion first discovered by Borrmann ${ }^{2,3}$ during his studies of the X-ray diffraction in perfect crystals. (For the quantum mechanical justification of the imaginary part of the crystal potential see Yoshioka ${ }^{4}$ or, e. g., the review article by Kambe and Molière ${ }^{5}$.)

If the wave function of the elastically scattered fast electrons inside a perfect crystal is expressed in terms of Bloch waves the solution of the Schrödinger equation can be reduced to the solution of an eigenvalue equation which is Hermitian for crystals without absorption and which becomes nonHermitian when absorption is taken into account by an appropriate imaginary part of the crystal potential. Since, in general, the imaginary part of the potential is small compared with the real part, it is usually assumed that the non-Hermitian eigen- 
value equation can be solved with sufficient accuracy by an incomplete first order perturbation treatment (Hirsch, Howie, Nicholson, Pashley and Whelan ${ }^{6}$, Metherell and Fisher ${ }^{7}$ ). By this treatment the Bloch functions of the absorption-free crystal are multiplied by absorption factors whereas the orthogonality of the Bloch functions remains unchanged.

The interpretation of transmission electron micrographs from lattice defects in crystalline specimens requires the calculation of the diffraction of fast electrons in imperfect crystals. For this purpose mainly two types of differential equations are used in the literature: (i) The differential equations of plane wave type (PW-eqs.) (Howie and Whelan ${ }^{8}$, Takagi $\left.{ }^{9}\right)$; (ii) the differential equations of Bloch wave type (BW-eqs.) (Howie ${ }^{10}$, Wilkens ${ }^{11}$, Howie and Basinski ${ }^{12}$ ). Both of these differential equations are derived from the Schrödinger equation in a similar manner. Consequently they are exactly equivalent when absorption is neglected *. However, if absorption is included similar problems to those in the case of perfect crystals arise. For this reason some authors treated the non-Hermitian eigenvalue equation for the derivation of the BW-eqs. in the same incomplete first order approximation as mentioned above, e. g. ${ }^{10,12}$, Cockayne ${ }^{14}$, Hirsch and Humphreys ${ }^{15}$. On the other hand, in ${ }^{11}$ a better degree of approximation was implicitly assumed, although its relation to the non-Hermitian eigenvalue equation was not clearly recognized.

In the present paper we will show that the equivalence of the PW-eqs. and the BW-eqs. is only preserved with a more rigorous solution of the eigenvalue equation. It will further be shown that the simple approximation described above is in fact sufficient for lattice defects with screened longrange strain fields (e.g., small dislocation loops) whereas for defects with unscreened long-range strain fields (e.g., dislocations) a better approximation is required which, however, destroys the orthogonality of the Bloch waves.

\section{Definitions}

For the following it is convenient to write a number of equations in matrix form. In general, a matrix $\boldsymbol{M}$ is constituted by its elements $M_{i k}$. The

\footnotetext{
* The differential equations using so-called "modified Blochwaves" ${ }^{13}$, which are less suitable for numerical computations, will be mentioned briefly in Sect. 9 and 10 .
}

horizontal and the vertical columns of the matrix are labelled by the subscripts $i$ and $k$, respectively. A diagonal matrix with the elements $a_{k}$ will be denoted by $\mathbf{D}\left[\alpha_{k}\right]$ with $D_{i k}=\delta_{i k} \cdot a_{k}, \delta_{i k}=\operatorname{Krod}$ ecker symbol. A vertical column vector will be written as $\left\{v_{n}\right\}$ or $\left\{w^{l}\right\}$ with $n$ or $l$ being the running index of the vector components.

We consider a crystal foil of thickness $t$. The $N$ reciprocal lattice vectors $\mathbf{g}_{n}$ (including $\mathbf{g}_{0}=0$ ) which are involved in the $N$-beam case lie parallel to the foil plane. The $z$-axis of a cartesian coordinate system points from the upper foil surface $(z=0)$ into the foil. A plane wave $\psi=\psi_{0}$ with

$$
\psi_{0}=\exp 2 \pi i \mathbf{k}_{0} \mathbf{r}
$$

is propagating from $z<0$ into the foil. The wave vector $\mathbf{k}_{0}$ is related to the total energy $E$ of the electron wave by

$$
E=h^{2} k_{0}{ }^{2} / 2 m
$$

with $h=$ Planck's constant and $m=$ electron mass.

\section{The PW-Equations}

In the description of the PW-eqs. the wave field $\psi$ at the depth $z=t$ (i. e. just below the lower foil surface) is described by a linear combination of $N$ plane waves $\psi_{n}$ propagating into the empty space below the crystal foil. $\psi$ and $\psi_{n}$ are given by

$$
\begin{gathered}
\psi=\sum_{n} \chi_{n}(t) \psi_{n}, \\
\psi_{n}=\exp 2 \pi i\left(\mathbf{k}_{0} \mathbf{r}+\mathbf{g}_{n} \mathbf{r}+s_{n} z\right) .
\end{gathered}
$$

Here $s_{n}$ is the excitation error of the reflexion $\mathbf{g}_{n}$ with respect to the direction of the wave vector $\mathbf{k}_{0}$ of the incident beam. In the column approximation (Hirsch, Howie and Whelan ${ }^{16}$ ) the amplitudes $\chi_{n}(t)$ of the plane waves $\psi_{n}$ are calculated by means of the PW-eqs. ${ }^{8,} 9$

$$
\begin{array}{r}
\mathrm{d}\left\{\chi_{n}(z)\right\} / \mathrm{d} z=2 \pi i \boldsymbol{D}\left[\exp -2 \pi i\left(s_{n} z+u_{n}(z)\right)\right] \\
\cdot \boldsymbol{U} \cdot \mathbf{D}\left[\exp 2 \pi i\left(s_{n^{\prime}} z+u_{n^{\prime}}(z)\right)\right] \cdot\left\{\chi_{n^{\prime}}(z)\right\} .
\end{array}
$$

The following abbreviations are used:

$$
u_{n}=\left(\mathbf{g}_{n} \mathbf{R}\right) .
$$

$\mathbf{R}=\mathbf{R}(z)=$ atomic displacement field vector along a given integration column which is characterized by the lateral coordinates $x$ and $y$. The elements $U_{n, n^{\prime}}$ of the matrix $\boldsymbol{U}$ are given by

$$
U_{n, n^{\prime}}=\left(-k_{0} e / 2 E\right) V_{n-n^{\prime}}
$$


where $e$ denotes the positive elementary charge and $V_{n}$ the Fourier coefficient of the crystal lattice potential $V(\mathbf{r})$ which is given by

$$
V(\mathbf{r})=\sum_{n} V_{n} \exp \left\{2 \pi i\left(\mathbf{g}_{n} \cdot \mathbf{r}\right)\right\}<0 .
$$

(The difference $n-n^{\prime}$ in the subscript on the right hand side of Eq. (3.5) stands for $\mathbf{g}_{n}-\mathbf{g}_{n^{\prime}}$.)

For a crystal with absorption the poitential $V(\mathbf{r})$ and, as a consequence, the matrix $\boldsymbol{U}$ is split into two parts,

$$
V(\mathbf{r})=\tilde{V}(\mathbf{r})+i V^{\prime}(\mathbf{r}), \quad \boldsymbol{U}=\tilde{\boldsymbol{U}}+i \boldsymbol{U}^{\prime}
$$

with $\tilde{\boldsymbol{U}}$ and $\boldsymbol{U}^{\prime}$ being Hermitian. The components of $\tilde{\boldsymbol{U}}$ and $\boldsymbol{U}^{\prime}$ are related to the corresponding two-beam extinction lengths $\xi$ and two-beam absorption lengths $\xi^{\prime}$, respectively, by

$$
\left|\tilde{U}_{n, n^{\prime}}\right|=1 / 2 \xi_{n-n^{\prime}},\left|U_{n, n^{\prime}}^{\prime}\right|=1 / 2 \xi_{n-n^{\prime}} .
$$

Equation (3.3) must be solved for $z=t$ with the boundary condition at $z=0$ :

$$
\left\{\chi_{n}(0)\right\}=\left\{\delta_{0, n}\right\} .
$$

\section{The BW-Equations}

In the BW description the solution $\psi$ at the depth $z$ inside the foil is represented by a linear combination of $N$ Bloch waves $b^{l}$ which are appropriately chosen with respect to the boundary condition at $\mathrm{z}=0$.

$$
\psi=\sum_{l} \varphi^{l}(\boldsymbol{z}) \cdot b^{l}\left(\mathbf{k}_{\mathbf{0}}, z\right) .
$$

For an imperfect crystal the Bloch waves $b^{l}$ are defined by ( see $^{11}$ )

$$
\begin{array}{r}
b^{l}\left(\mathbf{k}_{0}, z\right) \\
=\sum_{n} c_{n}{ }^{l} \exp \left\{2 \pi i\left(\mathbf{k}_{0} \mathbf{r}+\Delta k^{l} z+\mathbf{g}_{n} \mathbf{r}-u_{n}\right)\right\}
\end{array}
$$

with the normalization

$$
\sum_{n} c_{n}^{l}\left(c_{n}^{l}\right)^{*}=1, \quad * \text { conjugate complex. }
$$

The so-called "Anpassung" $\Delta k^{l}$ (Bethe ${ }^{17}$ ) is determined as the eigenvalue of index $l$ of the eigenvalue equation

$$
\left(\boldsymbol{U}+\boldsymbol{D}\left[s_{n}\right]\right)\left\{c_{n}\right\}=\Delta k\left\{c_{n}\right\} .
$$

The $c_{n}{ }^{l}$ are the components of the corresponding eigenvector $\left\{c_{n}\right\}^{l}$. We introduce a matrix $\boldsymbol{C}$ with the components $C_{n, l}=c_{n}{ }^{l}$. Then the complete set of the $N$ solutions of Eq. (4.4) is represented by

$$
\left(\boldsymbol{U}+\boldsymbol{D}\left[s_{n}\right]\right) \cdot \boldsymbol{C}-\boldsymbol{C} \cdot \boldsymbol{D}\left[\Delta k^{l}\right]=0 .
$$

In ${ }^{11}$ it was shown that within the column approximation ${ }^{16}$ the (weakly $z$-dependent) Bloch amplitudes $\varphi^{l}(z)$ are determined by the following set of differential equations:

$$
\begin{array}{r}
\boldsymbol{C} \cdot \boldsymbol{D}\left[\exp 2 \pi i \Delta k^{l} z\right] \mathrm{d}\left\{\varphi^{l}(z)\right\} / \mathrm{d} z=2 \pi i \boldsymbol{D}\left[u_{n, z}\right] \\
\cdot \boldsymbol{C} \cdot \boldsymbol{D}\left[\exp 2 \pi i \Delta k^{l^{\prime}} z\right] \cdot\left\{\varphi^{l^{\prime}}\right\}
\end{array}
$$

with the abbreviation

$$
u_{n, z}=\mathrm{d} u_{n} / d z .
$$

Making use of an inverse matrix $\boldsymbol{C}^{-1}$ defined by

$$
\boldsymbol{C}^{-1} \cdot \boldsymbol{C}=\boldsymbol{E}=\text { unity matrix }
$$

the differential Eqs. (4.6) can be rearranged into the $\mathrm{BW}$-eqs.

$$
\begin{aligned}
\mathrm{d}\left\{\varphi^{l}\right\} / \mathrm{d} z= & 2 \pi i \boldsymbol{D}\left[\exp -2 \pi i \Delta k^{l} z\right] \\
& \cdot \boldsymbol{Q}_{z} \cdot \boldsymbol{D}\left[\exp 2 \pi i \Delta k^{l^{\prime}} z\right] \cdot\left\{\varphi^{l^{\prime}}\right\}
\end{aligned}
$$

with

$$
\boldsymbol{Q}=\boldsymbol{C}^{-1} \cdot \boldsymbol{D}\left[u_{n}\right] \cdot \boldsymbol{C}, \boldsymbol{Q}_{z}=\mathrm{d} \boldsymbol{Q} / \mathrm{d} z .
$$

The BW-eqs. must be solved for $z=t$ with the boundary conditions

$\left\{\varphi^{l}(0)\right\}=\boldsymbol{C}^{-1} \cdot \boldsymbol{D}\left[\exp 2 \pi i u_{n}(0)\right] \cdot\left\{\delta_{0, l}\right\}$.

If $\left\{\varphi^{l}(t)\right\}$ is the solution at $z=t$ the desired amplitudes $\left\{\chi_{n}(t)\right\}$ of the plane waves $\psi_{n}$ below the lower foil surface are obtained from Eqs. (3.1) and (4.1). After some simple algebraic operations we find

$$
\begin{aligned}
\chi_{n}(t)=\boldsymbol{D}\left[\exp -2 \pi i\left(s_{n} t+u_{n}(t)\right)\right] \\
\cdot \boldsymbol{C} \cdot \boldsymbol{D}\left[\exp 2 \pi i \Delta k^{l} t\right] \cdot\left\{\varphi^{l}(t)\right\} .
\end{aligned}
$$

\section{Derivation of the BW-Eqs. from the PW-Eqs.}

We replace in Eq. (4.12) the argument $t$ by the coordinate $z$. Then, by differentiation of Eq. (4.12) with respect to $z$ and by comparison with the PW-eqs. (3.3) we arrive after some rearrangements at

$$
\begin{aligned}
& \mathrm{d}\left\{\varphi^{l}\right\} / \mathrm{d} z=2 \pi i \cdot \boldsymbol{D}\left[\exp -2 \pi i \Delta k^{l} z\right] \\
& \cdot \boldsymbol{C}^{-1} \cdot\left(\boldsymbol{D}\left[u_{n, z}\right] \cdot \boldsymbol{C}+\left\langle\left(\boldsymbol{U}+\boldsymbol{D}\left[s_{n}\right]\right) \cdot \boldsymbol{C}\right.\right. \\
&\left.\left.-\boldsymbol{C} \cdot \boldsymbol{D}\left[\Delta k^{\prime}\right]\right\rangle\right) \cdot \boldsymbol{D}\left[\exp 2 \pi i \Delta k^{l^{\prime}} z\right] \cdot\left\{\varphi^{l^{\prime}}\right\} .
\end{aligned}
$$

Obviously, this equation differs from Eq. (4.9) only by the term which is enclosed by \langle\rangle . This term, however, is identical with the left side of the eigenvalue Equation (4.5). From this result we conclude that the $\mathrm{PW}$-eqs. and the $\mathrm{BW}$-eqs. are identical when the eigenvalue equation is fulfilled. 


\section{Solution of the Eigenvalue Equation}

In this section we solve the eigenvalue Eq. (4.5) in different steps of approximation.

Approach (i): If absorption is neglected the matrix $\boldsymbol{U}=\tilde{\boldsymbol{U}}$ is Hermitian. The corresponding solution of Eq. (4.5) will be characterized by a tilde and will be used in the following as the "zero order" solution

$$
\Delta k^{l}=\Delta \tilde{k}^{l}, c_{n}^{l}=\tilde{c_{n}}{ }^{l} .
$$

In this case the matrix $\tilde{\boldsymbol{C}}$ (elements $\tilde{C}_{n, l}=\tilde{c}_{n}^{l}$ ) is unitary with

$$
\tilde{\boldsymbol{C}}^{-1}=\tilde{\boldsymbol{C}}^{+}=\text {adjungate matrix to } \boldsymbol{C} .
$$

Consequently the eigenvectors $\left\{c_{n}\right\}^{l}$ are orthogonal.

If absorption is included, the matric $\boldsymbol{U}$ is given by Eq. (3.7). Considering $i \boldsymbol{U}^{\prime}$ as a small correction to $\tilde{\boldsymbol{U}}$ Eq. (4.5) can be solved by a perturbation calculation. Accordingly we write

$$
\Delta k^{l}=\tilde{\Delta k^{l}}+i \tilde{q^{l}}, \quad c_{n}^{l}={\tilde{c_{n}}}^{l}+i \Delta \tilde{\Delta c_{n}^{l}}
$$

where $\tilde{q}^{l}$ and $\tilde{\Delta c_{n}^{l}}$ are assumed to be small corrections. Inserting this trial solution into Eq. (4.5) one obtains $\Delta \tilde{k^{l}}$ and $\tilde{c}_{n}^{l}$ as the zero order solutions again. The terms which are linear in either $\tilde{q}^{l}, \Delta \tilde{c}_{n}^{l}$, or $\boldsymbol{U}^{\prime}$ govern the first order solution.

Approach (ii): In an incomplete first order solution (which is usually applied to the electron diffraction in perfect lattices ${ }^{6,} \boldsymbol{7}$ ) only the corrections of the eigenvalues are considered:

$$
\tilde{q}^{l}=\sum_{n, n^{\prime}}\left(\tilde{c}_{n}^{l}\right)^{*} U_{n, n^{\prime}}^{\prime} \tilde{c}_{n}^{l}, \Delta \tilde{c}_{n}{ }^{l}=0 .
$$

Consequently in this approach the property of the Bloch functions as a set of orthogonal functions remains unchanged. [If only the so-called normal absorption is considered, i. e., if $U_{n, n^{\prime}}^{\prime}=U_{0}{ }^{\prime} \cdot \delta_{n, n^{\prime}}$, the solution in Eq. (6.4) is exact.]

Approach (iii): If the first order solution of Eq. (4.5) is completed we find $\tilde{q}^{l}$ as given above and

or in matrix form

$$
\Delta \tilde{c_{n}}{ }^{l}=\sum_{l^{\prime}} \tilde{c}_{n}^{l^{\prime}} A_{l^{\prime} l}
$$

$$
\boldsymbol{C}=\tilde{\boldsymbol{C}}+i \Delta \tilde{\boldsymbol{C}}, \quad \Delta \tilde{\boldsymbol{C}}=\tilde{\boldsymbol{C}} \cdot \boldsymbol{A} .
$$

$\boldsymbol{A}$ is a "scew-Hermitian" matrix with the elements

$$
\begin{aligned}
& A_{l^{\prime}, l}=-\frac{\sum_{n, n^{\prime}}\left(\tilde{c}_{n}^{l^{\prime}}\right)^{*} \cdot U_{n, n^{\prime}}^{\prime} \cdot \tilde{c}_{n^{\prime}}^{l}}{\Delta \tilde{k}^{l^{\prime}}-\Delta \tilde{k}^{l}} \text { for } l \neq l^{\prime}, \\
& =0 \\
& \text { for } l=l^{\prime} \text {. }
\end{aligned}
$$

The orthogonality of the eigenvectors is now violated in linear terms of $\boldsymbol{A}$ : With $\boldsymbol{A}=-\boldsymbol{A}^{+}$and Eq. (6.6) it follows

$$
\begin{aligned}
\boldsymbol{C}^{+} \cdot \boldsymbol{C}=(\tilde{\boldsymbol{C}}+i \Delta \tilde{\boldsymbol{C}})^{+} \cdot & (\tilde{\boldsymbol{C}}+i \Delta \tilde{\boldsymbol{C}}) \\
= & \boldsymbol{E}+2 i \boldsymbol{A}-\boldsymbol{A} \cdot \boldsymbol{A} .
\end{aligned}
$$

An approximate representation of the inverse matrix $C^{-1}$ is given by

$$
\boldsymbol{C}^{-1}=(\tilde{\boldsymbol{C}}+i \Delta \tilde{\boldsymbol{C}})^{-1} \approx\left(\tilde{\boldsymbol{C}}^{+}-i \boldsymbol{A} \tilde{\boldsymbol{C}}^{+}\right)
$$

which leads to

$$
\boldsymbol{C}^{-1} \cdot \boldsymbol{C}=\boldsymbol{E}+\boldsymbol{A} \cdot \boldsymbol{A} .
$$

Approach (iv): Eq. (4.5) can be solved exactly with the results denoted by

$$
\begin{gathered}
\Delta k^{l}=\Delta \hat{k}^{l}+i \hat{q}^{l}, \\
c_{n}^{l}=\hat{c}_{n}^{l}+i \Delta \hat{c}_{n}^{l}, \text { or } \boldsymbol{C}=\hat{\boldsymbol{C}}+i \Delta \hat{\boldsymbol{C}}
\end{gathered}
$$

where $\Delta \hat{k^{l}}, \hat{q^{l}},{\hat{c_{n}}}^{l}$ and $\Delta{\hat{c_{n}}}^{l}$ are real.

With respect to the inverse matrix $\boldsymbol{C}^{-1}$ we consider only centrosymmetric crystals. In this case the matrices $\tilde{\boldsymbol{U}}$ and $\boldsymbol{U}^{\prime}$ can be chosen real and symmetric. Multiplying Eq. (4.5) from both sides with $C^{-1}$ yields

$$
\boldsymbol{C}^{-1} \cdot\left(\boldsymbol{U}+\boldsymbol{D}\left[s_{n}\right]\right)=\boldsymbol{D}\left[\Delta k^{l}\right] \cdot \boldsymbol{C}^{-1} .
$$

In comparison to Eq. (4.8), this is an alternative definition of $\boldsymbol{C}^{-1}$. In terms of the exact solution, Eq. (4.5) is written as

$$
\begin{aligned}
\left(\tilde{\boldsymbol{U}}+\boldsymbol{D}\left[s_{n}\right]\right. & \left.+i \mathbf{U}^{\prime}\right) \cdot(\hat{\boldsymbol{C}}+i \Delta \hat{\boldsymbol{C}}) \\
& =(\hat{\boldsymbol{C}}+i \Delta \hat{\boldsymbol{C}}) \cdot \boldsymbol{D}\left[\Delta \hat{k}^{l}+i \hat{q}^{l}\right] .
\end{aligned}
$$

Now we make use of the assumption that $\tilde{\boldsymbol{U}}$ and $\boldsymbol{U}^{\prime}$ are real and symmetric: Going to the conjugate complex form and then to the adjungate matrices we arrive at

$$
\begin{aligned}
\left(\tilde{\boldsymbol{C}}^{+}+i \Delta \hat{\boldsymbol{C}}^{+}\right) \cdot\left(\tilde{\boldsymbol{U}}+\boldsymbol{D}\left[s_{n}\right]+i \boldsymbol{U}^{\prime}\right) \\
=\boldsymbol{D}\left[\Delta \hat{k}^{l}+i \hat{q}^{l}\right] \cdot\left(\hat{\boldsymbol{C}}^{+}+i \Delta \hat{\boldsymbol{C}}^{+}\right) .
\end{aligned}
$$

Comparing with Eq. (6.11) we conclude that under the assumption made the inverse matrix $\boldsymbol{C}^{-1}$ is given by

$$
\boldsymbol{C}^{-1}=\hat{\boldsymbol{C}}^{+}+i \Delta \hat{\boldsymbol{C}}^{+} .
$$

In the approaches (i) and (iv) the eigenvalue equation is exactly fulfilled. In these cases, according to Sect. 5 , the PW-eqs. and the BW-eqs. are exactly equivalent. However, in the approaches (ii) and (iii) both types of differential equations 
differ from each other to the same extent to which the eigenvalue equation is not fulfilled. From this comparison we conclude that for crystals showing anomalous absorption the $\mathrm{PW}$-eqs. are equivalent to the exact solution of the eigenvalue equation. It should be noted that this particular problem does not appear explicitly in the derivation of the PWeqs. from the Schrödinger equation ${ }^{9}$.

\section{Intraband and Interband Scattering}

From now on we restrict ourselves to centrosymmetric crystals. Then the matrix $\boldsymbol{C}$ can be chosen real. As a consequence, for the approaches (i) and (ii) of Sect. 6 the matrix $\boldsymbol{Q}$ in Eq. (4.10), as calculated with $\boldsymbol{C}=\tilde{\boldsymbol{C}}$, is real and symmetric. For the approach (iii) we write

$$
\mathbf{Q}=\tilde{\mathbf{Q}}+i \Delta \tilde{\mathbf{Q}} .
$$

In linear terms of $\boldsymbol{A}$ the (real and symmetric) correction $\Delta \tilde{\mathbf{Q}}$ is given by

$$
\Delta \boldsymbol{Q}=\tilde{\mathbf{Q}} \cdot \boldsymbol{A}-\boldsymbol{A} \cdot \tilde{\mathbf{Q}} .
$$

In the case of approach (iv) the matrix $\boldsymbol{Q}$ can be split in a similar way into a real and an imaginary part,

$$
\boldsymbol{Q}=\hat{\boldsymbol{Q}}+i \Delta \hat{\mathbf{Q}} .
$$

The dependence of the imaginary correction terms $i \Delta Q^{l, l^{\prime}}$ on the direction of incidence of the primary wave will be considered in detail elsewhere ${ }^{18}$. Here it should be mentioned that the correction terms are comparatively small (in the two beam case even zero) if for one of the reciprocal lattice vectors $\mathbf{g}_{n}$ the Bragg condition is exactly fulfilled. This follows from the symmetry properties of the eigenvectors $\left\{c_{n}\right\}^{l}$ under this condition (cf. ${ }^{7}$ ).

For convenience Eq. (4.9) is rewritten as a sum

$$
\begin{aligned}
\mathrm{d}\left\{\varphi^{l}\right\} / \mathrm{d} z=2 \pi i \sum_{l^{\prime}} Q_{z}^{l, l^{\prime}} \\
\cdot \varphi^{l^{\prime}} \exp \left\{2 \pi i\left(\Delta k^{l^{\prime}}-\Delta k^{l}\right) z\right\} .
\end{aligned}
$$

The diagonal terms $\left(l=l^{\prime}\right)$ and the off-diagonal terms $\left(l \neq l^{\prime}\right)$ on the right hand side represent the "intraband" scattering and the "interband" scattering, respectively. For the separation of these two parts of scattering we consider an integration column which is sufficiently far away from the centre of the lattice defect. "Sufficiently far away" means that the interband scattering can be neglected. This is the case if the condition

$$
\int Q_{z}^{l, l^{\prime}} \exp \left\{2 \pi i\left(\Delta k^{l^{\prime}}-\Delta k^{l}\right) z\right\} \mathrm{d} z \approx 0, \text { for } l \neq l^{\prime},
$$

is fulfilled. Then Eq. (7.4) degenerates into

$$
\mathrm{d} \varphi^{l} / \mathrm{d} z \approx 2 \pi i Q_{z}^{l, l} \cdot \varphi^{l} .
$$

Integration gives

$$
\varphi^{l}(z) \approx \varphi^{l}(0) \exp \left\{2 \pi i\left[Q^{l, l}(z)-Q^{l, l}(0)\right]\right\}
$$

with $\varphi^{l}(0)$ given by the boundary condition Equation (4.11). We define "phase factors" $P_{ \pm}^{l}(z)$ according to

$$
P_{ \pm}^{l}(z)=\exp \left\{ \pm 2 \pi i\left[Q^{l, l}(z)-Q^{l, l}(0)\right]\right\}
$$

and introduce auxiliary functions $\Phi^{l}(z)$ defined by

$$
\varphi^{l}(z)=\Phi^{l}(z) \cdot P_{+}^{l}(z) .
$$

Substituting $\varphi^{l}(z)$ by $\Phi^{l}(z)$ in Eq. (7.4) we obtain a modified form of the BW-eqs. which is free of interband scattering (see also ${ }^{11}$ )

$$
\begin{aligned}
\mathrm{d}\left\{\Phi^{l}(z)\right\} / \mathrm{d} z=2 \pi i \sum_{l^{\prime} \neq l} Q_{z}^{l . l^{\prime}} \\
\cdot \Phi^{l^{\prime}}(z) \cdot P_{+}^{l^{\prime}}(z) \cdot P_{-}^{l}(z) \cdot \exp \left\{2 \pi i\left(\Delta k^{l^{\prime}}-\Delta k^{l}\right) z\right\}
\end{aligned}
$$

and which must be solved with the boundary conditions

$$
\Phi^{l}(0)=\varphi^{l}(0) .
$$

If $\Phi^{l}(t)$ represents the appropriate solution of these equations at $z=t$, the amplitudes $\varphi^{l}(t)$ needed for the calculation of the plane wave amplitudes $\chi_{n}(t)$ are given by

$$
\varphi^{l}(t)=\Phi^{l}(t) \cdot P^{l}+(t) .
$$

If only intraband scattering occurs Eq. (7.12) degenerates to

$$
\varphi^{l}(t)=\varphi^{l}(0) \cdot P^{l}+(t) .
$$

Because of the elimination of the intraband scattering Eq. (7.10) is more suitable for a first Born approximation than Eq. (7.4) (compare also ${ }^{11}$ ). To this purpose we substitute on the right hand side of Eq. (7.10) the functions $\Phi^{l^{\prime}}(z)$, which take the intraband scattering into account, by their values at $z=0$ :

$$
\Phi^{l^{\prime}}(z) \approx \Phi^{l^{\prime}}(0)=\varphi^{l^{\prime}}(0)
$$

(compare also ${ }^{15}$ ). This leads to

$$
\begin{gathered}
\varphi^{l}(t)=\varphi^{l}(0) \cdot P_{+}^{l}(t)+2 \pi i P_{+}^{l}(t) \sum_{l^{\prime} \neq l} \varphi^{l^{\prime}}(0) \int_{0}^{t} Q_{z}^{l, l^{\prime}} \\
\cdot P_{+}^{l^{\prime}}(z) P_{-}^{l}(z) \cdot \exp \left\{2 \pi i\left(\Delta k^{l^{\prime}}-\Delta k^{l}\right) z \mathrm{~d} z\right\} .
\end{gathered}
$$


Recently, Cockayne ${ }^{14}$ has used Eq. (7.10) for a first Born approximation by setting on the right side

$$
\Phi^{l^{\prime}}(z) \approx \varphi^{l^{\prime}}(0) \cdot P_{-}^{l^{\prime}}(z) \text {, or } \varphi^{l^{\prime}}(z)=\varphi^{l^{\prime}}(0) \text {. }
$$

This treatment by which the term $P_{+}^{l^{\prime}}(z)$ under the integral of Eq. (7.15) is omitted, is obviously not consistent with Eqs. (7.7) and (7.13).

The approaches (ii) and (iii) are the most interesting ones among the solution approaches of the eigenvalue equation described in Section 6. (Approach (i) is unrealistic since absorption is neglected; in general, approach (iv) is not expected to be significantly different from approach (iii).) As the essential point we realize that in approach (ii) the components $\tilde{c}_{n}^{l}$ of the eigenvectors and, consequently, the matrix elements $Q^{l, l^{\prime}}$ are not modified by the absorption. On the other hand, using approach (iii) the imaginary correction terms $i \tilde{\Delta c}_{n}{ }^{l}$ and $i \Delta \tilde{Q}^{l, l^{\prime}}$ enter into the intensities $\left|\chi_{n}(t)\right|^{2}$ of the plane waves $\psi_{n}$ in different ways: in the matrix $\boldsymbol{C}$ in Eq. (4.12) where the $\chi_{n}(t)$ are calculated from the $\varphi^{l}(t)$ and in the prefactors $Q_{z}^{l, l^{\prime}}$ of the BW-eqs. in Eq. (7.4). With respect to the latter case it should be stated that the correction of the diagonal terms, $i \Delta \tilde{Q}^{l, l^{\prime}}$, appears in the exponents of the phase factors of the modified Equation (7.10).

\section{Results of Comparative Contrast Calculations}

For a number of defect configurations (single dislocations and narrow dislocation dipoles) the contrast profiles were calculated using either approach (ii), (iii) or (iv). Some representative examples are shown in Figs. 1 to 3. In all cases the plotted intensities,

$$
I_{n}(x) \propto\left|\chi_{n}(t, x)\right|^{2},
$$

are normalized to the background intensities. The superscripts $\mathrm{r}$ or c at $I_{n}$ refer to "real eigenvectors" (approach (ii)) and "complex eigenvectors" (approach (iii) or (iv)), respectively. (For the considered potentials no significant differences were found between the curves calculated with approach (iii) and (iv).) We used the displacement field of straight dislocations in an infinite and isotropic material. The dislocations are assumed to lie parallel to the $y$-axis at the positions $x=x_{0}$ and $z=z_{0}$. The direction of incidence of the primary wave is characterized either by the normalized excitation error $w=s_{1} \xi_{1}$ of the first order reflexion $\mathbf{g}_{1}$ or, in a more general way, by the parameter $E$ introduced in ${ }^{19}$ : For a systematic $N$-beam case, $E=n$ ( $=$ integer) means that the reflexion $\mathbf{g}_{n}$ is exactly excited. For the two-beam case $(N=2)$ we assumed $\xi_{1} / \xi_{1}{ }^{\prime}=$ 0.1 and $\xi_{0}{ }^{\prime}=\xi_{1}{ }^{\prime}$. For $N=4 \quad(-1 \leqq n \leqq 2)$ or $N=6 \quad(-2 \leqq n \leqq 3)$ the Fourier coefficients of the complex potential of $\mathrm{Cu}$ as tabulated by Radi ${ }^{20}$ were used.

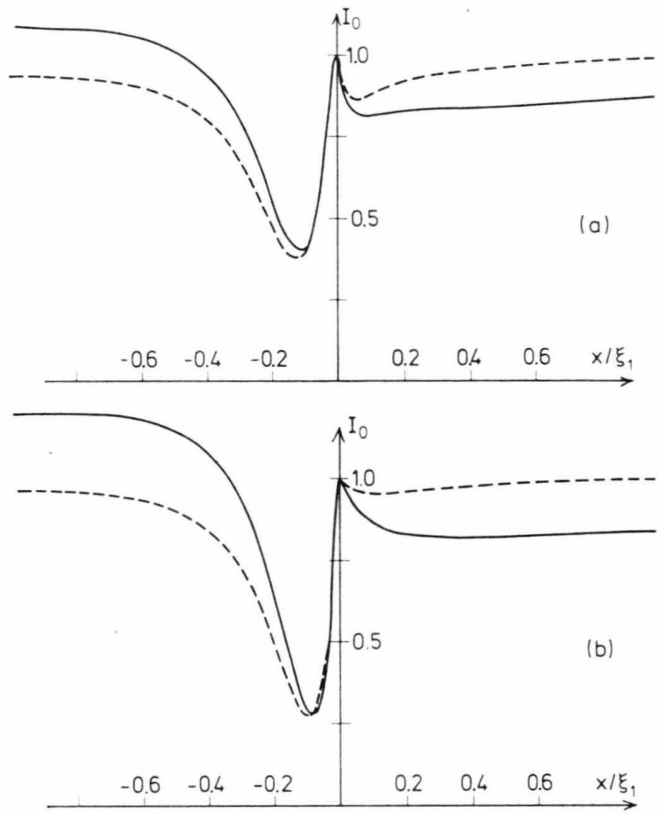

Fig. 1. Computed bright field images of a screw dislocation for the two-beam case. $\mathbf{g}_{1} \cdot \mathbf{b}=2, t=8 \xi_{1}$; position of the dislocation: $x_{0}=0, z_{0}=t / 2 . \xi_{1} / \xi_{1}{ }^{\prime}=0.1$. In (a) $w=0.3(E=1.06)$, in (b) $w=0.6(E=1.12)$. The intensities $I_{0} \mathrm{c}$ and $I_{0} \mathrm{r}$ are shown as solid and broken lines, respectively.

(1) Single dislocations, Figure 1. For $N=2$ and $w=0 \quad(E=1)$ the imaginary correction terms $i \Delta c_{n}{ }^{l}$ are zero. Consequently, in this case there is no difference between $I_{n}^{r}$ and $I_{n}{ }^{c}$. With increasing $w$ the profiles $I_{0}{ }^{c}$ reveal pronounced long-range contrast tails with $I_{0}{ }^{c}>1$ for $x<0$ and $I_{0}{ }^{c}<1$ for $x>0$, cf. Figure 1. For the corresponding profiles $I_{0}{ }^{r}$ the long-range contrast tails are essentially weaker. For instance, for $w=0.6$ we have $1 I_{0}{ }^{c}=$ $I_{0}{ }^{c}(x=+\xi)-I_{0}{ }^{c}(x=-\xi)=-0.36$, whereas the corresponding value of $\Delta I_{0}{ }^{r}$ is only -0.03 . According to Fig. 1 the curves of $I_{0}{ }^{c}$ and $I_{0}{ }^{r}$ deviate significantly from each other already at the flanks of the deep contrast minima. Consequently, the half widths of the (negative) peaks are broader for the $I_{0}{ }^{r}$-curves. However, the depths and the positions of 
the minima are practically the same in both cases.

Similar results were obtained for $I_{1}$. Furthermore, the characteristic differences between $I_{n}{ }^{r}$ and $I_{n}{ }^{c}$ do not depend on the choice of $N$.

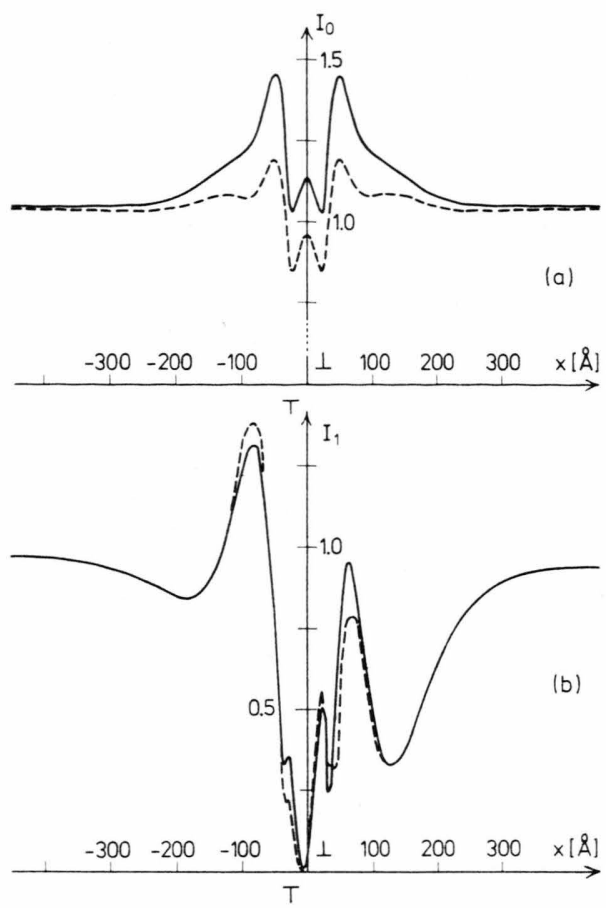

Fig. 2. Computed bright field (a) and dark field (b) images of a dipole of edge dislocations in copper. Positions of the dislocations: $x_{0}=-25 \AA, z_{0}=t / 2+25 \AA$ and $x_{0}=+25 \AA$, $z_{0}=t / 2-25 \AA$. Further parameters are: Voltage $=100 \mathrm{kV}$, $N=4, \mathbf{g}_{1} \cdot \mathbf{b}=2, \mathbf{g}_{1}=(220), t=1500 \AA, E=0.8(w=-1.02)$. The intensities $I_{n}^{\mathrm{c}}$ and $I_{n}^{\mathrm{r}}$ are shown in solid and broken lines, respectively.

(2) Dislocation dipoles, Figure 2. The tails of both contrast profiles, $I_{n}{ }^{r}$ and $I_{n}{ }^{c}$, coincide quite well. In the inner part of the profiles the positions of the contrast maxima and minima are the same in both modes of calculations. However, there are some remarkable differences (up to 25\%) in the heights of the contrast extrema. For other excitation errors and larger foil thicknesses the differences are smaller.

(3) Dark field contrast $I_{-1}$ corresponding to the weakly excited reflexion $\mathbf{g}_{-1}$. For the both examples shown in Fig. 3 we find similar results as in the cases (1) and (2): In Fig. 3 a (pair of dislocations of the same sign) there are some differences in the long-range contrast tails which are not found in Fig. $3 \mathrm{~b}$ (dislocation dipole). The heights of the contrast peaks are slightly different. However, we

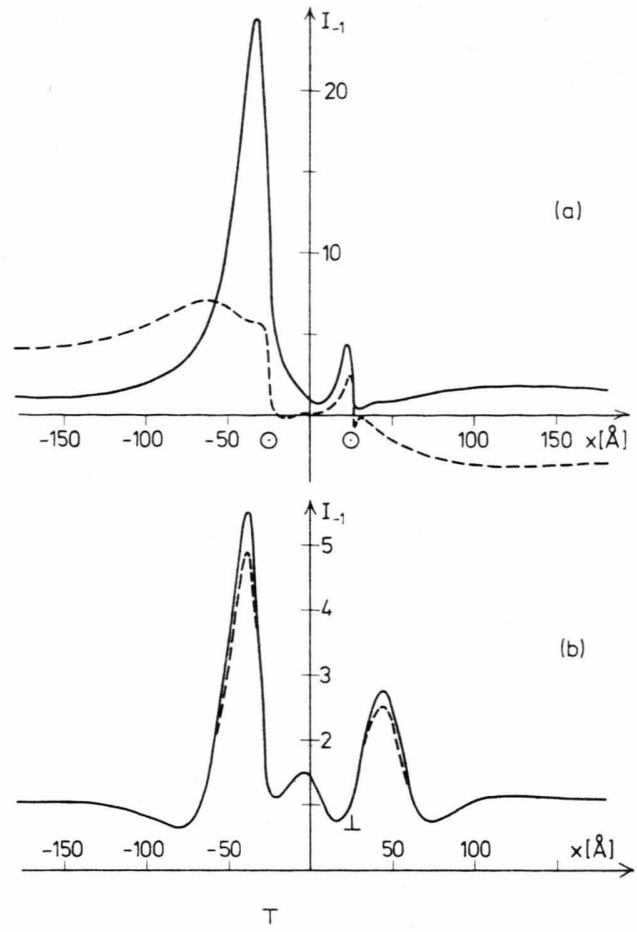

Fig. 3. Computed weak-beam images taken with $\mathbf{g}_{-1}$. The intensity $I_{-1}^{\mathbf{c}}$ is shown in solid lines. (a) Pair of screw dislocations. Positions of the dislocations: $x_{0}=-25 \AA, z_{0}=t / 2$ and $x_{0}=+25 \AA, z_{0}=t / 2$. Voltage $=100 \mathrm{kV}, N=6, \mathbf{g}_{1} \cdot \mathbf{b}=1$, $\mathbf{g}_{1}=(200), t=2680 \AA, E=1.4 \quad(w=0.76)$. The broken line show ten times enlarged the difference $I_{-1}^{\mathrm{c}}-I_{-1}^{\mathrm{r}}$. (b) Dipole show ten times enlarged the difference $I^{\mathrm{c}}-1-I_{-1}{ }_{-1}$ (b) Dipole of edge dislocations. The parameters are the same as in Figure 2 . The broken line indicates the intensity $I_{-1}^{\mathrm{r}}$.

state that also for the weak-beam contrast profiles the peak positions are not shifted significantly by the mode of calculations.

\section{Discussion of the Contrast Calculations}

The results of Sect. 8 show that three different features of the contrast profiles can be considered separately: (i) The long-range contrast tails, (ii) the heights and widths of the main peaks, and (iii) the positions of the peaks. Obviously, the mode of calculation (" $r$ " or " $c$ ") has only a minor or even negligible influence on (ii) and (iii). Therefore we now restrict ourselves to a brief discussion of point (i).

Long-range contrast tails from defects with an unscreened long-range strain field (e.g., single dislocations, pairs of dislocations of the same sign, etc.) are well known, cf., e. g., the contrast calcula- 
tions of Howie and Whelan ${ }^{21}$, which are based on the $\mathrm{PW}$-eqs. For the experimentalists this particular contrast phenomenon represents a valuable tool for the determination of the sign of the Burgers vectors of the dislocations.

The results of this paper demonstrate that in terms of the BW-eqs. the long-range contrast tails are only calculated when the eigenvectors are corrected by means of approaches (iii) or (iv). Extensive theoretical studies of the experimental conditions of the long-range contrast tails were performed by Wilkens, Rühle and Häussermann ${ }^{22}$. They applied to this problem the concept of modified Bloch waves" $13, *$. Comparing the results of 22 with the concept of "conventional" Bloch waves as discussed in the present paper it can be concluded that the long-range contrast tails are closely related to the intraband scattering or - in the notation of the present paper - to the intraband term $P^{l}{ }_{+}(\mathrm{t})$ in Eq. (7.12). For instance, the displacement field vector $\mathbf{R}$ of a single dislocation in the middle of the foil results at the foil surfaces in

$$
\mathbf{g}_{n} \mathbf{R}(t)-\mathbf{g}_{n} \mathbf{R}(0) \approx \pm \frac{1}{2} \mathbf{g}_{n} \mathbf{b}
$$

$(\mathbf{b}=$ Burgers vector of the dislocation) where the signs on the right side of Eq. (9.1) are valid for opposite sides of the contrast profiles. Consequently, the imaginary part of $Q^{l, l}(t)-Q^{l, l}(0)$ in the exponent of Eq. (7.8) calculated with the correction term $i \Delta c_{n}{ }^{l}$ of the approaches (iii) or (iv) leads to

$$
P^{l}{ }_{+}(t)<1 \text { and } P_{+}^{l}(t)>1 \text {, respectively. }
$$

These inequalities are directly responsible for the occurrence of the long-range contrast tails.

Since the long-range contrast tails are well established experimentally we must conclude that the comparatively simple approach (ii) which was applied in $10,12,14,15$ is insufficient concerning the entire contrast profile. However, if only the positions of the contrast peaks are considered, approach (ii) can be used. This result which is relevant for the application of the weak-beam method ${ }^{14}$ can

* Modified Bloch waves differ from the Bloch waves used in the present paper in so far as they are not related to a perfect reference lattice: In the eigenvalue equation of modified Bloch waves the excitation errors $s_{n}$ are functions of the local orientation of the lattice. Consequently, in a distorted crystal the eigenvalues and the orthogonal eigenvectors are functions of $\mathbf{r}$. This means that the "tie point" of a modified Bloch wave on the corresponding dispersion branch is not fixed throughout the crystal, as assumed in the present paper, but moves on the branch according to the local lattice orientation. be understood by a more detailed discussion of the $\mathrm{BW}$-eqs: In the region of the contrast profiles sufficiently close to the image position of the dislocation cores the contribution of the intraband scattering is masked to a large extend by the interband scattering. The latter is, obviously, much less sensitive to the correction of the eigenvectors.

The case of lattice defects with a screened longrange strain field (e.g., narrow dislocation dipoles or small dislocation loops) will be discussed briefly. For integration columns passing outside the dipole (loop) through the foil the displacements at the foil surfaces are nearly compensated due to the opposite signs of the dislocations,

$$
\mathbf{g}_{n} \mathbf{R}(t)-\mathbf{g}_{n} \mathbf{R}(0) \approx 0,
$$

which means that in the "outside" region no significant difference between $I_{n}{ }^{r}$ and $I_{n}{ }^{c}$ is expected, cf. Fig. 2 and $3 \mathrm{~b}$. On the other hand, for integration columns passing through the dipole (loop) Eq. (9.3) must be substituted by

$$
\mathbf{g}_{n} \mathbf{R}(t)-\mathbf{g}_{n} \mathbf{R}(0) \approx \pm \mathbf{g}_{n} \mathbf{b} .
$$

The corresponding intraband scattering is, obviously, negligible with respect to the peak positions, cf. Figs. 2 and 3 and the preceding paragraph.

\section{Final Remarks}

In the present paper we have expressed the differential equations of Bloch-wave type (BW-eqs.) using different approaches to the non-Hermitian eigenvalue equation. In approach (i) the imaginary part of the crystal potential is neglected (zero order solution). Consequently this approach has no practical applications. In approach (ii), which is generally applied to the diffraction in perfect crystals, the eigenvalues (wave vectors of the Bloch waves) are corrected by an incomplete first order perturbation calculation. In this approximation the wave vectors are complemented by an imaginary part which is responsible for the anomalous absorption (Borrmann effect), whereas the eigenvectors (amplitude coefficients of the partial waves of the Bloch waves) remain unchanged. Hence in this approach the orthogonality of the Bloch waves is retained. In approach (iii) also the eigenvectors are corrected by the first order perturbation oalculation giving rise to additional imaginary terms. Finally, in approach (iv), the non-Hermitian eigenvalue equation is assumed to be exactly solved irrespective of what the 
quantum mechanical interpretation of this solution is (cf., e. g., Sprague and Wilkens ${ }^{23}$ ). In the latter two cases the orthogonality of the corrected Bloch waves is violated in linear (or higher order) terms of the imaginary part of the crystal potential. (Since this imaginary part is usually assumed to be small compared with the real part the differences between the approaches (iii) and (iv) are in general negligible.)

In order to estimate which mode of approach is necessary for the calculation of the diffraction contrast from a particular lattice defect, a number of comparative contrast calculations were performed using either approach (ii) or (iv) (or (iii), cf. Setion 9).

Especially for single dislocations imaged with a non-zero excitation error pronounced differences were found in the flanks and the tails of the contrast profiles, where the contrast is predominantly determined by the intraband scattering, of. Sect. 7 and 9. Long-range contrast tails which are wellknown experimentally are only obtained when corrected eigenvectors are used (approach (iii) or (iv) ). On the other hand the central part of the contrast profiles - especially the position of the contrast maxima or minima - are less sensitive to the mode of approach.

Narrow dislocation dipoles and other defect configurations with screened long-range strain fields (e. g., small dislocation loops) do not give rise to long-range contrast tails. Accordingly, for these defect configurations the contrast profiles calculated in either of the approximations (ii) to (iv) show only small differences which may be hard to detect experimentally.

The phenomenon of long-range contrast tails from single dislocations indicate clearly that, in principle, the BW-eqs. should be used with corrected eigenvalues and eigenvectors. As already mentioned above, in this case the orthogonality of the Bloch waves is seriously violated. Therefore we must con-

* In ${ }^{19}$, in which the contrast from small spherical inclusions and dislocation loops was calculated, approach (ii) was used. In the other papers of Rühle, Wilkens and co-workers concerned with similar problems the calculations were performed with approach (iv). This was not stated explicitely.

1 H. Hashimoto, A. Howie, and M. J. Whelan, Proc. Roy. Soc. London A 269, 80 [1962].

2 G. Borrmann, Z. Physik 42, 157 [1941].

3 G. Borrmann, Z. Physik 127, 297 [1950].

${ }^{4}$ H. J. Yoshioka, J. Phys. Soc. Japan 12, 618 [1957]. clude that for distorted crystals with anomalous absorption the concept of Bloch waves as defined in a perfect reference crystal becomes doubtful as soon as one considers the physical significance of the properties of individual Bloch waves. Nevertheless, for the calculation of the diffraction contrast this concept has proved to be very successful and convenient for numerical computations as well as for analytical first order perturbation calculations ${ }^{19}, 24,25, * *$.

Let us briefly return to the modified Bloch waves mentioned in Section 9. Without going into details, it should be pointed out that the differential equations of type Eqs. (4.9) or (7.4), when expressed in a similar way in terms of modified Bloch waves (cf. ${ }^{13}$ ) do not contain diagonal elements. This means that for distorted crystals showing anomalous absorption the imaginary correction terms of the eigenvector components do not enter in a direct way into the phase factors of the differential equations. This is contrary to the "conventional" BW-eqs., cf. Section 7. Consequently, in terms of modified Bloch waves, the long-range contrast tails from single dislocations as the most significant criterion for the necessity of a correction of the eigenvectors are appropriately calculated already with the same incomplete first order solution of the eigenvalue equation (approach (ii)) in which the orthogonality of the modified Bloch waves is retained (cf. ${ }^{22}$ ). This result confirms from quite a different point of view earlier statements ${ }^{13}$ that for distorted crystals modified Bloch waves are physically more significant than "conventional" Bloch waves defined in a perfect reference crystal.

\section{Acknowledgement}

The authors are indebted to Prof. Dr. A. Seeger and Dr. H. Teichler for critical comments. The assistence of Dr. H. Mughrabi for the preparation of the manuscript is gratefully acknowledged.

5 K. Kambe and K. Molière, in "Advances in Structure Research by Diffraction Methods" (R. Brill and R. Mason, editors), Pergamon Press, New York 1970, p. 53.

6 P. B. Hirsch, A. Howie, R. B. Nicholson, D. W. Pashley, and M. J. Whelan, Electron Microscopy of Thin Crystals, Butterworths, London 1965.

7 A. J. F. Metherell and R. M. Fisher, Phys. Stat. Sol. 32, 551 [1969].

8 A. Howie and M. J. Whelan, Proc, Roy. Soc. London A 263, 217 [1961].

9 S. Takagi, Acta Cryst. 15, 1311 [1962]. 
10 A. Howie, Proc. Roy. Soc. London A 271, 268 [1963].

11 M. Wilkens, Phys. Stat. Sol. 6, 939 [1964].

12 A. Howie and Z. S. Basinski, Phil. Mag. 17, 1039 [1968].

13 M. Wilkens, Phys. Stat. Sol. 13, 529 [1966].

14 D. J. H. Cockayne, Z. Naturforsch. 27 a, 452 [1972].

15 P. B. Hirsch and C. J. Humphreys, Proc. 5th Europ. Regional Conf. on Electron Microscopy, Manchester, p. 520 (1972).

16 P. B. Hirsch, A. Howie, and M. J. Whelan, Phil. Trans. Roy. Soc. A 252, 499 [1960].

17 H. Bethe, Ann. Phys. 87, 55 [1928].

18 K.-H. Katerbau, to be published.

19 F. Häussermann, K.-H. Katerbau, M. Rühle, and M. Wil- kens, J. Microscopy, in press.

20 G. Radi, Acta Cryst. A 26, 41 [1970].

21 A. Howie and M. J. Whelan, Proc. Roy. Soc. London A 267, 206 [1962].

22 M. Wilkens, M. Rühle, and F. Häussermann, Phys. Stat. Sol. 22, 689 [1967].

23 J. A. Sprague and M. Wilkens, Proc. 7th Intern. Congress for Electron Microscopy, Grenoble, Vol. I, p. 95 (1970).

24 M. Wilkens and M. Rühle, Phys. Stat. Sol. (b) 49, 749 [1972].

25 F. Häussermann, M. Rühle, and M. Wilkens, Phys. Stat. Sol. (b) 50, 445 [1972].

\title{
An Exact 2-Point Distribution for Burger's Turbulence *
}

\author{
P. Gräff, H. Tasso, and J. Steuerwald \\ Max-Planck-Institut für Plasmaphysik, 8046 Garching bei München, Federal Republic of Germany
}

(Z. Naturforsch. 28 a, 690-692 [1973]; received 6 February 1973)

\begin{abstract}
An exact possible turbulence for Burger's equation is found. It allows the 2-point distribution function to be calculated in closed form.
\end{abstract}

Burger's equation offers a solvable model of hydrodynamics. This would be of great value to strong turbulence as a means of testing approximation procedures or numerical methods if the statistical initial value problem could also be treated. However, rigorous results are difficult to obtain since one is dealing with a Liouville equation for the infinitely many degrees of freedom of a continuum. (A rigorous description of this kind of problem was first given by $\operatorname{Hopf}^{1}$ 1952.) The special case for the spatial white noise at $t=0$ was sketched earlier ${ }^{2}$. An admissible 1-point distribution for all times has already been found ${ }^{3}$. It is of Lorentz form:

$$
P_{1}(v, t) \approx 1 /\left[a(t)+b(t) v^{2}\right] .
$$

In this paper we derive the 2-point distribution function in closed form from the Hopf characteristic functional.

Burger's equation is of the form:

$$
\partial v / \partial t+v \partial v / \partial x-v \partial^{2} v / \partial x^{2}=0 .
$$

The ansatz $v=-2 v \partial \ln |M| / \partial x$ due to Cole reduces Eq. (1) to the heat conduction equation:

Reprint requests to Dr. L. Johannsen, Bibliothek, MaxPlanck-Institut für Plasmaphysik, D-8046 Garching bei München.

$$
\partial M / \partial t=v \partial^{2} M / \partial x^{2}
$$

whose relevant solutions are

$$
\begin{aligned}
M(x, t)= & (1 / \sqrt{2 \pi t v}) \int_{-\infty}^{+\infty} \\
& \cdot \exp \left\{-(x-\xi)^{2} / 2 t \nu\right\} M_{0}(\xi) \mathrm{d} \xi
\end{aligned}
$$

and

$$
\begin{aligned}
p(x, t) & =\left\langle M_{1} M_{2}\right\rangle=(1 / \sqrt{8 \pi t} v) \\
& \int_{-\infty}^{+\infty} \exp \left\{-(x-\xi)^{2} / 8 t v\right\} p(\xi) \mathrm{d} \xi .
\end{aligned}
$$

Consider the characteristic functional ${ }^{1}$ for a Gaussian measure which is conserved by the heat equation. For the simultaneous measure of $M$ and $M^{\prime}$ we get

$$
\begin{gathered}
\varphi_{M, M^{\prime}}(\alpha, \beta)=\varepsilon\left[\operatorname { e x p } \left\{i \int_{-\infty}^{+\infty} \alpha(x) M(x) \mathrm{d} x\right.\right. \\
\left.\left.+i \int_{-\infty}^{+\infty} \beta(x) M^{\prime} \mathrm{d} x\right\}\right] \\
\left.=\varepsilon\left[\exp _{-\infty}^{+\infty}\left(\alpha-\beta^{\prime}\right) M(x) \mathrm{d} x\right\}\right]=\varphi_{M}\left(\alpha-\beta^{\prime}\right)
\end{gathered}
$$

* This work was performed under the terms of the agreement on association between the Max Planck-Institut für Plasmaphysik and EURATOM. 